\title{
The advantage of being left-handed in interactive sports
}

\author{
Norbert HaGemanN \\ University of Münster, Münster, Germany
}

\begin{abstract}
As compared with their prevalence in the general population, left-handers are overrepresented in the expert domain of many interactive sports. This study examined to what extent this is due to negative perceptual frequency effects - that is, whether the greater frequency of tennis matches with right-handed opponents makes it possible to discriminate the stroke movements of right-handed players more precisely. Fifty-four right-handed and 54 left-handed males in three equal-sized groups of varying levels of tennis expertise (national league experts, local league intermediates, and novices) completed a tennis anticipation test in which they had to predict the subsequent direction of an opponent's temporally occluded tennis strokes on a computer screen. The results showed that all three groups were better at predicting the direction of strokes by right-handed players. This supports the hypothesis that the overrepresentation of left-handers in the expert domain is partly due to perceptual frequency effects.
\end{abstract}

With slight geographical variations, the proportion of left-handers in the population seems to have hardly changed for several thousands of years (Faurie \& Raymond, 2004). Depending on which activity is observed (e.g., throwing, using a hammer), it ranges from about 10\% to 13\% (Raymond, Pontier, Dufour, \& Møller, 1996). However, among top-level international athletes, left-handers ${ }^{1}$ are overrepresented (as compared with their prevalence in the general population) in most interactive sports in which two or more athletes play or fight each other directly (e.g., tennis, fencing, or boxing), but not in noninteractive or individual sports (e.g., golf or swimming) (Grouios, 2004; Grouios, Tsorbatzoudis, Alexandris, \& Barkoukis, 2000; Raymond et al., 1996). For example, in an analysis of world rankings from 1968 to 1999, Holtzen (2000) showed that lefthanders were clearly overrepresented among the world's top 10 ranking players in tennis (male, $24.06 \%$; female, $11.80 \%$ ), the world's number one ranking players (male, $34.4 \%$; female, $30.3 \%$ ), and Grand Slam finalists (male, $22.27 \%$; female, $18.75 \%$ ). A higher proportion of lefthanders can also be confirmed in the top ranks of other sports, such as fencing (Bisiacchi, Ripoll, Stein, Simonet, \& Azemar, 1985), baseball (Coren, 1993; Goldstein \& Young, 1996), and cricket (Aggleton \& Wood, 1990; Edwards \& Beaton, 1996; Wood \& Aggleton, 1989).

\section{Earlier Explanations}

Two explanations for this phenomenon are discussed particularly frequently in the literature (Grouios, 2004; Grouios et al., 2000). The first assumes that left-handers possess a neuropsychological advantage (the innate superiority hypothesis); the second, that they have a strategic or tactical advantage (the strategic advantage hypothesis).
Innate superiority hypothesis. Geschwind and Galaburda (1987) pointed to the developmentally determined enlargement of brain regions in the right hemisphere of left-handers, arguing that this should particularly favor them when performing activities placing demands on the right half of the brain, such as spatial imagery tasks (e.g., Rossi \& Zani, 1986) or tasks requiring certain attentional functions (Bisiacchi et al., 1985). Bisiacchi et al. confirmed an advantage for left-handed fencers on several attentional tasks, suspecting that the right half of their brain is neuroanatomically highly suitable for such activities.

Taddei, Viggiano, and Mecacci (1991) reported similar findings in fencing. They studied 8 male fencers (with at least 10 years of fencing experience) and 8 controls (college students). One half of each group were left-handers according to the Edinburgh Handedness Inventory (EHI; Oldfield, 1971). Using psychophysical measures (visual evoked potentials), they found that visual stimuli evoked potentials more rapidly in left-handed fencers than in right-handed fencers and that this effect was particularly marked for large visual stimuli.

Particularly with regard to motor skills, one significant factor seems to be whether the motor task is performed with one hand (e.g., darts or bowling) or both hands (e.g., golf, ice hockey, or baseball). When performing a task with both hands, left-handers could benefit from the weaker lateralization of the two brain hemispheres (Grouios, 2004). The stronger nondominant side or the superior interplay between the two sides could then lead to better overall performance in bimanual activities. For example, McLean and Ciurczak (1982) concluded that this weaker lateralization was responsible for the superior average batting performance of left-handed baseball 
players (who both pitch and bat with their left hands), as compared with right-handed players who also bat with their left hands (but pitch with their right).

Grondin, Guiard, Ivry, and Korens's (1999) analysis of the batting performance of all major league baseball players (1871-1992) also produced the same finding. However, these authors interpreted it as being at least partially a consequence of the specialization of both hands, in the sense of Guiard's (1987) model of a kinematic chain. This model posits that the preferred and nonpreferred hands have complementary functions, with optimal performance requiring both hands to be used in a synchronized sequence.

Strategic advantage hypothesis. Other discussions have focused on a strategic or tactical advantage of lefthanders, as compared with right-handers (Grouios, 2004; Grouios, Koidou, Tsorbatzouidis, \& Alexandris, 2002). These assume that the unfamiliar playing strategies and patterns of attack in left-handers are particularly advantageous in interactive sports (Faurie \& Raymond, 2005). Because players become used to the hitting patterns or playing style of right-handed opponents, attacks from the opposite side in, for example, boxing or fencing take them by surprise (Coren, 1993). In addition to this surprise effect, the motor responses to such an attack may also be underpracticed. Because this type of attack by left-handers is less frequent, defensive reactions are less automatic and, therefore, possibly less effective.

Edwards and Beaton (1996) used strategic factors to explain the frequency distribution of left-handed bowlers in cricket across four seasons between 1981 and 1991. They compared the frequency of two different types of bowlers (seam and spin bowlers), who differ in the degree of rotation they put into the ball, and found an overrepresentation of left-handed spin bowlers (more rotation), but not of seam bowlers. From a strategic perspective, the explanation for this finding is that it is easier for a left-handed spin bowler to produce a left-sided rotation of the ball. Such bowls will spin away from a right-handed batsman, making them harder to hit (see also Brooks, Bussière, Jennions, \& Hunt, 2004).

If left-handers enjoy a general advantage on motor tasks (particularly ones making high visuospatial demands) because of neuropsychological differences, as proposed by the innate superiority hypothesis, this advantage should also be found in other types of sports. If not, this would tend to favor the strategic effect. Aggleton and Wood (1990; see also Wood \& Aggleton, 1989) studied this through comparisons with sports corresponding to this profile that simultaneously reveal no strategic-tactical advantages: snooker, darts, 10-pin bowling, golf, and soccer (goalkeepers). None of these sports revealed any overrepresentation of left-handers. Grouios et al. (2000) applied the same approach to study the distribution of handedness in highly skilled athletes in interactive $(n=576)$ and noninteractive $(n=536)$ sports. They found a higher probability for the occurrence of left-handers in interactive sports such as boxing, fencing, tennis, and basketball, but not in individual sports such as cycling, gymnastics, or swimming.

\section{Perception of Human Movements}

The discussion on the strategic advantages of lefthanders neglects one essential aspect. Particularly in interactive sports, performance depends on sport-type-specific perceptual or anticipatory skills (Williams, Davids, \& Williams, 1999). In interactive sports, the ability to exploit the movement information available during early stages of an action sequence (e.g., postural orientation) and anticipate an opponent's intentions is crucial to successful performance and permits a better preparation of one's own motor reaction (for an overview, see Williams et al., 1999). In this context, numerous expertise studies using the temporal occlusion paradigm have confirmed that experts can predict the direction of an opponent's action earlier and more precisely than novices. The typical experimental scenario has experts and novices viewing videotape sequences that present the opponent from the perspective of the responding player. The videotapes stop at a set timepoint (e.g., when a racket makes contact with a ball), and the participant is asked to estimate the further direction of the action. Examples can be found for both tennis (e.g., Rowe \& McKenna, 2001; Ward, Williams, \& Bennett, 2002) and badminton (e.g., Abernethy \& Russell, 1987; Hagemann, Strauß, \& Cañal-Bruland, 2006).

Focus of orientation in the perception of human movements. Generally, people are very good at distinguishing different activities in their perceptions. Research using point-light displays reveals how well the perception system can recognize gender (e.g., Pollick, Lestou, Ryu, \& Cho, 2002), estimate weights during lifting movements (Runeson \& Frykholm, 1981), and distinguish between different sports techniques (Pollick, Fidopiastis, \& Braden, 2001). These studies assume that this sensitivity in distinguishing between different human movements depends on visual experience; in other words, if a pattern of motion occurs frequently, it will be easier to discriminate (cf. Giese \& Poggio, 2003; Jacobs, Pinto, \& Shiffrar, 2004).

On a neurophysiological level, computer tomography has shown that the posterior superior temporal sulcus (STS) is particularly active when individuals are perceiving human movements (Grossman et al., 2000). Electrophysiological studies using single probes in the STS also confirm the involvement of this region in the recognition of human movements (Oram \& Perrett, 1996). These have revealed that the majority of neurons in the STS react sensitively to specific body or body-part movements. Experimental manipulations of stimulus material have also revealed the high specificity of movement representation. For example, one cell in the anterior STS reacts to the right-sided profile of a person moving to the right. This same cell does not fire, however, when the left-sided profile of a person moves in the same direction (backward) or when the right-sided profile moves to the left (Oram \& Perrett, 1996).

This physiological specificity in visual movement perception is also reflected in psychological theories. For example, Freyd (1987) assumed that the dynamics of movements are an integral component of their mental representation. It can therefore be suspected that side-specific features are also 
mirrored in dynamic representations (see Olofsson, Nyberg, \& Nilsson, 1997). Support for this assumption comes from the side-specific priming effects reported by Daems and Verfaillie (1999, Experiments 2 and 3). Using longterm priming studies, these authors were able to show that the presentation of human body positions during a learning phase (images) will lead to faster recognition performance only when the presentations in the test phase take the same horizontal orientation (Experiments 2 and 3) or the same depth orientation (Experiments 1 and 4) (see also Verfaillie, 1993). These experiments show that human movements are stored in a perspective-dependent form and not as 3-D representations (e.g., Marr \& Nishihara, 1978; see also Stone, 1998,1999 , on the role of the direction of rotation in the recognition of 3-D objects).

Side-specific perception of human movements in sport. Because of this strong dependence on both visual experience and the horizontal orientation, it is proposed here that the sport-type-specific ability to perceive movement patterns carried out with the left hand is less well developed. This is due essentially to the lower frequency with which athletes in interactive sports are confronted with movements by left-handed opponents. I call this the negative perceptual frequency effect hypothesis.

McMorris and Colenso (1996) took the horizontal orientation of sports movements into account when studying the perceptual performance of 7 male professional soccer goalkeepers in England. Using the temporal occlusion paradigm, they presented 20 penalty kicks by 4 professional soccer players ( 5 kicks each by 2 left-footed and 2 rightfooted players) from the perspective of the goalkeeper. The video clips were displayed in random sequence, and the goalkeepers had to estimate where the ball would cross the goal line. Results showed that the directions of shots by the right-footed players were easier to recognize than those of the left-footed players. Post hoc interviews with the goalkeepers confirmed the suspicion that advance cues on the directions of shots by left-footed players are more difficult to perceive. Nonetheless, both the personspecific characteristics of the penalty takers and the small sample size greatly restrict the generalizability of these findings. Although all penalty takers were professional soccer players, it cannot be ruled out that the left-footed players were simply better at taking penalties. Moreover, other factors, such as the speed of the penalty shot, may have been involved.

The goal of the present study was to combat these methodological weaknesses and confirm experimentally that the sport-type-specific ability to perceive left-hand patterns of movement is less developed, as compared with right-hand patterns. This should test the negative perceptual frequency effect hypothesis and identify one further reason why left-handers are overrepresented in top-level interactive sports.

\section{METHOD}

\section{Participants}

A total of 108 males took part in this study. To control for the effect of own handedness, 54 right-handers (RH) and 54 left-handers
(LH) were selected with the German version of the EHI (Oldfield, 1971). ${ }^{2}$ These persons were then divided into three groups on the basis of their tennis-playing performance. There were 36 (18 righthanders, 18 left-handers) tennis players with professional ambitions who were playing in one of the four highest German tennis leagues at the time of the experiment (called experts in the following). This group had a mean age of 25.14 years $(S D=4.24)$. Its members had been playing tennis for an average of 18.86 years $(S D=3.98)$ and were training currently for an average of $11.39 \mathrm{~h}$ per week $(S D=$ 3.50 ). The second group of 36 participants (likewise, 18 righthanders, 18 left-handers) also had tennis experience in various local leagues (called intermediates in the following). They had a mean age of 24.14 years $(S D=4.21)$, had been playing tennis for an average of 15.83 years $(S D=3.63)$, and were training currently for an average of $6.17 \mathrm{~h}$ per week $(S D=2.71)$. The final group had no tennis experience ( $n=36 ; 18$ right-handers, 18 left-handers). It contained college students from different faculties (called novices in the following), with a mean age of 26 years $(S D=2.60)$.

\section{Test Procedure}

The participants viewed videotaped sequences of tennis strokes on a laptop computer and had to predict the direction and depth of each stroke. The videotapes showed tennis players carrying out a stroke from the perspective of the returning player (see Figure 1). The stroke movement was halted at the moment in time when the racket touched the ball. The participants had to report the direction and depth of the stroke in a schematic display of their own half of the tennis court (using the mouse with their dominant hand to click where the ball would land in the court). The dependent variable was the radial difference between the estimated point and the actual point where the ball had landed in the court (in meters).

The tennis players in the video clips were two left-handed and two right-handed members of a male team from the third highest tennis league in Germany. They had a mean age of 24 years and had been playing in their clubs for an average of 17.3 years at the time of the study. Twelve strokes (six groundstrokes, four volleys, and two serves) were recorded from each player. One half of the strokes played the ball from the left; the other half, from the right. The point of contact in the other half of the court was determined and documented by two independent judges. Tennis strokes were recorded with a Sony DCR-TRV950E digital video camera from a central position on the baseline at a height of $1.48 \mathrm{~m}$.

The 48 strokes were processed with the digital video-processing software Adobe Premiere 7.5, so that each clip ended when contact was made between the racket and the ball. The digital video processing then made it possible to mirror the video clips horizontally, so that the same movement could be viewed as a stroke by both a rightand a left-handed player. Using such mirrored video sequences eliminated the effects of handedness-specific movement patterns (i.e., differences in difficulty) that would emerge in a demonstration by real left- or right-handed players (McMorris \& Colenso, 1996).

The 96 video clips lasted from 1.2 to $4.2 \mathrm{sec}$ and were presented in a random sequence as four homogeneous blocks of 24 clips (two players with the same handedness; in the original or mirrored version). The sequence of four blocks was balanced across participants. Care was taken to ensure that the first two blocks did not present the same person in both the original and the mirrored versions. To avoid irritation, the participants were informed after the second block that they would now be shown the same clips again in the mirrored version.

The videos were integrated into a specially written software program. This presented the video clip with a resolution of $600 \times 480$ pixels in the upper left- or right-hand corner of a display, with the response field $(370 \times 470$ pixel $)$ on the opposite side (see Figure 1$)$. The left or right presentation was balanced across participants. They worked on the test alone and in quiet surroundings. Each participant completed five test trials before the actual test. Radial error was subjected to a 3 (group) $\times 2$ (handedness of participant $) \times 2$ (playing hand in video) $\times 2$ (initial handedness of player in video) mixed ANOVA. Alpha was set at .05, and effect sizes were calculated. 


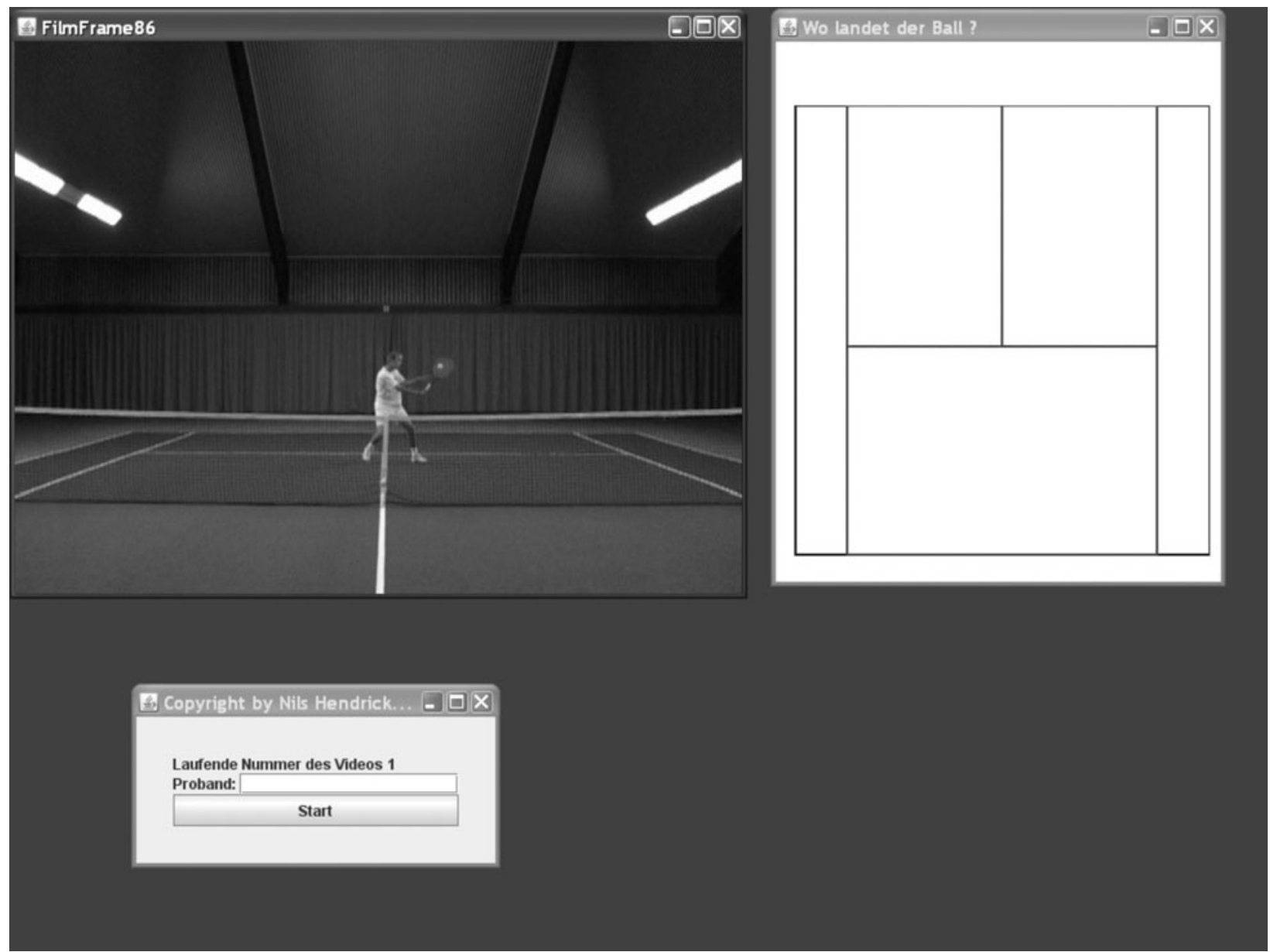

Figure 1. Screenshot of tennis anticipation test.

\section{RESULTS}

The mixed ANOVA revealed a significant main effect of group $\left[F(2,102)=38.62, p<.01, \eta_{\mathrm{p}}^{2}=.43\right]$ and a significant main effect of playing hand in video $[F(1,102)=$ 83.77, $\left.p<.01, \eta_{\mathrm{p}}^{2}=.45\right]$. Experts performed best of all, followed by intermediates, and then novices (all paired comparisons, $p<.001$ ). In all three groups, the directions of strokes by left-handers were more difficult to recognize than those of right-handers. The significant interaction between group and playing hand in video additionally showed that the differences between the prediction of left- and right-handed strokes varied across the three groups $\left[F(1,102)=3.78, p<.05, \eta_{\mathrm{p}}^{2}=.07\right.$; see Figure 2]. This difference was largest in the expert group $\left(M_{\text {diff }}=\right.$ $43.59 \mathrm{~cm})$.

To also estimate the radial error ${ }^{3}$ in relation to the probability of guessing, the interval-scaled horizontal deviations in centimeters were transformed into correct versus false decisions on the direction of the stroke (stroke to the left vs. stroke to the right). The mean correctness of decisions was markedly better than chance in all three groups (experts $=84.5 \%$, intermediates $=82.2 \%$, nov- ices $=74.0 \%$ ). This performance measure also revealed that the direction of strokes by a left-hander was harder to predict than that of a right-hander $[F(1,102)=50.22$, $p<.01, \eta_{\mathrm{p}}^{2}=.33$; experts, right-handers $=87.5 \%$, lefthanders $=81.5 \%$; intermediates, right-handers $=83.7 \%$, left-handers $=80.8 \%$; novices, right-handers $=76.7 \%$, left-handers $=71.3 \%]$.

Taking the participants' handedness into account revealed the same pattern regardless of group membership: Both left- and right-handers were better at predicting the direction of right-handed strokes. Nonetheless, the interaction between playing hand in video and handedness of participant also revealed that left-handers seemed to possess an additional advantage when it came to predicting right-handers $\left[F(1,102)=4.01, p<.05, \eta_{\mathrm{p}}^{2}=.04\right.$; see Figure 3].

By mirroring the video material, each player could be presented as either a left- or a right-hander. This manipulation made it possible to test whether it would be easier to predict the two left- or right-handers independently from the handedness presented in the video clip. When the video material was categorized in terms of the original playing hand, a main effect of initial handedness of player 


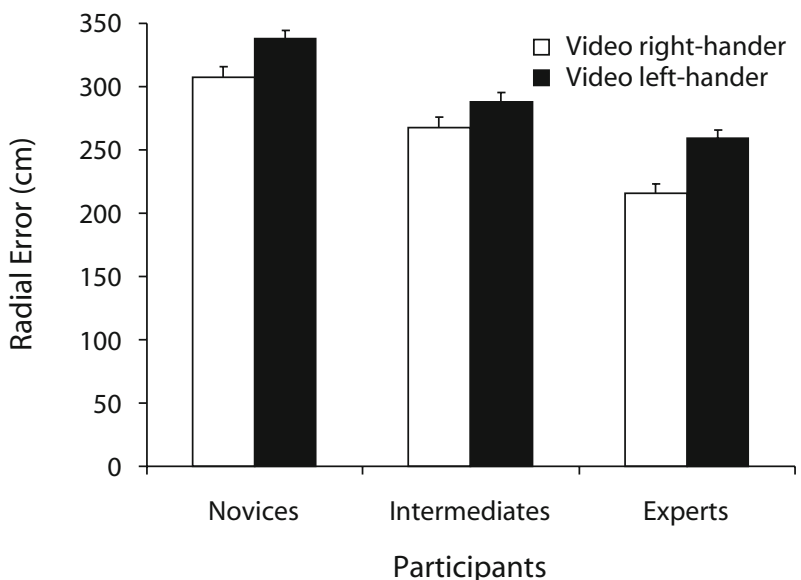

Figure 2. Radial prediction error $( \pm S E)$ for left- and righthanded tennis strokes as a function of level of expertise.

in video showed that it was easier to predict the strokes carried out by the two right-handed players $\left[M_{\text {diff }}=\right.$ $\left.7.34 \mathrm{~cm} ; F(1,102)=4.57, p<.05, \eta_{\mathrm{p}}^{2}=.43\right]$.

This effect was accompanied by an unexpected interaction between playing hand in video and initial handedness of player in video $\left[F(1,102)=6.82, p<.05, \eta_{\mathrm{p}}^{2}=.06\right.$; see Figure 4]. Although the video clips of left-handers (both the mirrored right-handers and the initial left-handers) produced almost identical results, the strokes by the original right-handers were easier to predict than those of the mirrored left-handers.

Comparing the initial handedness of the players in the video clips with the expertise levels and the handedness of the participants revealed a three-way interaction $\left[F(2,102)=6.39, p<.01, \eta_{\mathrm{p}}^{2}=.11\right]$. This interaction could be summarized as follows: Although novices found it easy to predict the direction of the stroke in persons with whom they shared the same handedness (independently from the hand playing the stroke in the video clip), this effect was inverted in experts. The experts were better at predicting the directions of strokes by persons with the opposite handedness independently of the handedness presented in the video clip.

\section{DISCUSSION}

The starting point for this study was the frequently confirmed overrepresentation of left-handers in top-level interactive sports. The perceptual frequency effect was introduced as one previously neglected possible reason for this increased prevalence. The study used the example of tennis to test whether the ability to discriminate lefthanded movement patterns is less well developed than that for right-handed movement patterns. It confirmed that tennis strokes performed with the right hand are easier to discriminate; that is, the direction of the stroke can be predicted more reliably. The very high effect size additionally indicates that this effect is highly significant.

Early recognition of the direction of a move is highly relevant for athletes in interactive sports. Because of the high speed of play (e.g., tennis serves that exceed $150 \mathrm{mph}$ ), athletes have to react very quickly, and an early recognition of the action planned by the opponent will give them a time advantage. A range of different temporal occlusion experiments have confirmed unanimously that experts are able to make much earlier and more precise decisions regarding the action intentions of their opponents. The temporal advantage of experts over novices in badminton, for example, ranges between 80 and $160 \mathrm{msec}$ (e.g., Abernethy \& Russell, 1987; Hagemann \& Strauß, 2006). This typical expertise effect could also be seen when levels of expertise were compared in the present study.

It is necessary to point out here that the motor reactions required in the present study, just like those reported in most other studies on visual perception in sports, do not correspond to those required in the real sport situation (mouse click vs. return in tennis). According to Milner and Goodale's (1995) model, these laboratory tasks basically make demands only on the ventral stream (vision for perception) and not on the dorsal stream (vision for action) of visual information processing. The ventral stream delivers information on the characteristics of objects and their relationships for the purpose of perceptual identification and classification. The dorsal stream, in contrast, is responsible ad hoc for the visual control of selected actions (Goodale \& Haffenden, 1998). In line with the findings in other studies (e.g., Farrow \& Abernethy, 2003), it is to be suspected that carrying out a test with a specific response pattern (in this case, strokes in tennis) should result in even greater differences between levels of expertise (for a discussion on the contribution of the ventral and dorsal systems to visual anticipation, see Abernethy \& Mann, 2008; van der Kamp, Rivas, van Doorn, \& Savelsbergh, 2008). Whether the differences between the perception of left- and right-handed strokes would also become larger is something that cannot be estimated on the basis of the findings reported here but will require further research.

The present study shows that the differences between the predictions of left- and right-handed tennis strokes do not decline with experience. One could have suspected

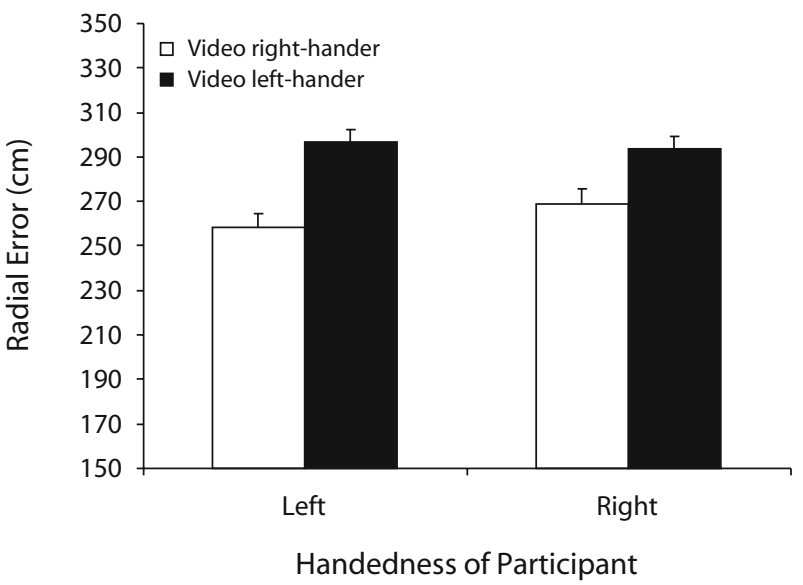

Figure 3. Radial prediction error $( \pm S E)$ for left- and righthanded tennis strokes as a function of participants' handedness. 


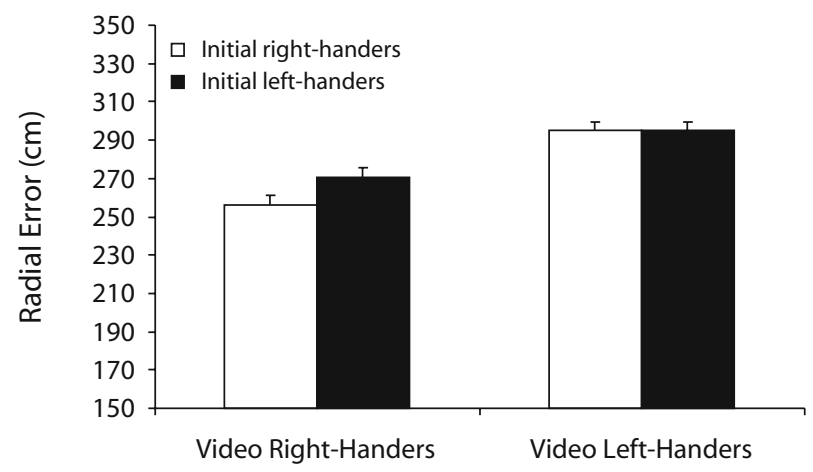

Player in the Video

Figure 4. Radial prediction error $( \pm S E)$ for left- and righthanded tennis strokes as a function of the initial handedness of the tennis strokes displayed.

that experience in playing with left-handers would also increase with greater expertise and that this would lead to a decline in the differences in the perception of left- and right-handers, particularly since left-handers are overrepresented at higher performance levels. Indeed, the greatest difference is found in the experts. This may be due to simple summation effects; in other words, more intensive training with right-handers (ca. $80 \%-90 \%$ of training time over 19 years) seems to have led to a greater consolidation of differences. Although more left-handers may be found at higher performance levels as well, the absolute proportion of right-handers is still far larger, so that the perception of right-handed strokes is bound to be trained more intensively on higher performance levels as well. This is why this (negative) perceptual frequency effect offers a further explanation for the overrepresentation of left-handers in top-level sports that has been neglected in earlier discussions (Grouios, 2004; Holtzen, 2000).

However, even novices display the difference in predicting left- and right-handed tennis strokes, and this cannot be explained through tennis-specific experience. Nonetheless, this may still be due to the perceptual frequency effect. Not only in the leisure-time domain (e.g., in games with various types of rackets, such as shuttlecock or table tennis), but also when interpreting many gestures (e.g., shaking hands, pointing out directions), individuals are confronted more frequently with movements of the right hand (Jacobs et al., 2004).

The side-specific perceptual achievements that can no longer be attributed to person-specific motion characteristics because of the use of mirrored movement patterns (cf. McMorris \& Colenso, 1996) are in line with the specificity reported on both neurophysiological and psychological levels of explanation. Alongside the neurophysiological findings reported by, for example, Oram and Perrett (1996), who located direction-sensitive cells in the anterior STS, there are also psychological theories indicating that side-specific aspects of body posture (Daems \& Verfaillie, 1999; Olofsson et al., 1997) or directions of rotation (Stone, 1998, 1999) are reflected in dynamic representations.

\section{Does the Participants' Own Handedness Exert an Influence?}

Recent cognitive theories on action control have pointed repeatedly to the significance of prior motor experiences in the visual perception of human movements (e.g., Hommel, Müsseler, Aschersleben, \& Prinz, 2001). The neuropsychological basis for this mechanism could be so-called mirror neurons in the ventral premotor cortex that are active not only when human movements are observed and imagined, but also when they are performed (e.g., Gallese, Fadiga, Fogassi, \& Rizzolatti, 1996). In this context, Knoblich and Flach (2001), for example, have shown that people are better at recognizing the direction in which a dart has been thrown (video clips of throwing movement until the dart leaves the hand) when they judge recordings of their own throws, rather than the throws of strangers. These experiments show what a major influence motor competencies can exert on the perception of movement information (for an overview, see Rizzolatti \& Craighero, 2004; Viviani, 2002). This line of argument would suggest that the movement pattern of a left-hander might be easier for a left-hander to discriminate than for a right-hander (and vice versa). However, the data from the present study tend to contradict this. Frequency effects might also be involved here: Left-handers, who more frequently play against right-handers than do right-handers against righthanders, might be able to discriminate the movements of right-handers better. This finding tends to favor theories emphasizing a strong dependence on visual experience (e.g., Giese \& Poggio, 2003; Johansson, 1973). As Jacobs et al. (2004) pointed out,

According to this view, the elevated frequency with which human movement occurs in inherently social environments enhances visual sensitivity to human movement relative to less frequent motion events, such as trees blowing in the wind and balls bouncing. As such, observers may exhibit particularly high levels of visual sensitivity to human movement simply because they see so much of it. (p. 822)

\section{Do the Movement Patterns of Left-Handers Simply Mirror the Movements of Right-Handers?}

Comparing predictions for the video clips of the two right-handers with those of the two left-handers without taking the hand to be seen on the video clip into account reveals that it is easier to predict the strokes of the two right-handers. This finding can be explained through the different techniques applied by individual players. A post hoc analysis shows that the mean prediction performance for the four players varies by as much as $56 \mathrm{~cm}$; that is, the quality of the prediction of the direction of the stroke based on its preparation varies across players.

If the movement pattern of a left-hander is assumed to be an identical copy of the movement of a right-hander (only mirrored horizontally) and vice versa, there should also be no interaction between the playing hand in video and initial handedness of player in video variables. The 
fact that mirroring (particularly of left-handers) does not result in an identical prediction performance seems to indicate the presence of special characteristics in the movement patterns of left-handers that make them harder to predict. However, this statement possesses little power, because of the small number of demonstration persons. Further studies will address this issue by comparing kinematics and predictions directly in several persons. The same applies to the three-way interaction (group $\times$ initial handedness of player in video $\times$ handedness of participant). Although one may speculate that experts will improve their discrimination performance (regardless of which side is presented) as they gain experience with the opposite hand in the training process, here as well, our interpretation is based on an analysis of only four different stroke movements. Moreover, we cannot explain why the right-handed experts are better at predicting left-handed strokes from initial left-handers than left-handed strokes from initial right-handers.

In sum, this study has managed to identify one further factor that may help to account for the overrepresentation of left-handers in interactive sports. This can be called a negative perceptual frequency effect (see Faurie \& Raymond, 2005) and can be assigned to the set of strategic advantages of left-handers in sport, thus making the postulated influence of neuropsychological advantages of left-handers less probable. Further research should examine other frequency-dependent factors that could explain the emergence of an overrepresentation. These could include tactical decisions - that is, automated decision preferences that are generalized incorrectly from tactical play against right-handers. There could also be frequencydependent motor automatization processes. Stroke movements directed toward weak points in games against righthanders might be performed more precisely (e.g., from the backhand side) than when having to play toward the opposite side in games against left-handers.

This finding also underlines the importance of visual experience in the perception of human movements. Here as well, further experiments should vary the side specificity and the similarity to one's own movement representation by presenting, for example, one's own and another person's movements (Knoblich \& Flach, 2001), differently automatized movements (Jacobs et al., 2004), or different movement classes (Loula, Prasad, Harber, \& Shiffrar, 2005).

\section{AUTHOR NOTE}

I thank Arndt Ziegler for his untiring support in planning and carrying out this research project and gathering the large amount of data. Correspondence concerning this article should be addressed to N. Hagemann, Department of Sport Psychology, University of Münster, Horstmarer Landweg 62B, 48149 Münster, Germany (e-mail: n.hagemann@ uni-muenster.de)

\section{REFERENCES}

Abernethy, B., \& Mann, D. (2008). Dual pathways or dueling pathways for visual anticipation? A response to van der Kamp, Rivas, van Doorn \& Savelsbergh (2007). International Journal of Sport Psychology, 39, 136-141.

Abernethy, B., \& Russell, D. G. (1987). The relationship between expertise and visual search strategy in a racquet sport. Human Movement Science, 6, 283-319.

AgGleton, J. P., \& Wood, C. J. (1990). Is there a left-handed advantage in "ballistic" sports? International Journal of Sport Psychology, 21, 46-57.

Bisiacchi, P. S., Ripoll, H., Stein, J. F., Simonet, P., \& Azemar, G. (1985). Left-handedness in fencers: An attentional advantage? Perceptual \& Motor Skills, 61, 507-513.

Brooks, R., Bussière, L. F., Jennions, M. D., \& Hunt, J. (2004). Sinister strategies succeed at the cricket World Cup. Proceedings of the Royal Society of London B, 271, 64-66.

COREN, S. (1993). Left hander: Everything you need to know about lefthandedness. London: Murry.

Daems, A., \& Verfaillie, K. (1999). Viewpoint-dependent priming effects in the perception of human actions and body postures. Visual Cognition, 6, 665-693.

Edwards, S., \& BEATon, A. (1996). Howzat?! Why is there an overrepresentation of left-handed bowlers in professional cricket in the UK? Laterality, 1, 45-50.

Farrow, D., \& Abernethy, B. (2003). Do expertise and the degree of perception-action coupling affect natural anticipatory performance? Perception, 32, 1127-1139.

FAURIE, C., \& RAYMOND, M. (2004). Handedness frequency over more than ten thousand years. Proceedings of the Royal Society of London $B, \mathbf{2 7 1}, \mathrm{S} 43-\mathrm{S} 45$.

Faurie, C., \& Raymond, M. (2005). Handedness, homicide and negative frequency-dependent selection. Proceedings of the Royal Society of London B, 272, 25-28.

FreYd, J. J. (1987). Dynamic mental representations. Psychological Review, 94, 427-438.

Gallese, V., Fadiga, L., Fogassi, L., \& Rizzolatti, G. (1996). Action recognition in the premotor cortex. Brain, 119, 593-609.

Geschwind, N., \& Galaburda, A. (1987). Cerebral lateralization: Biological mechanisms, associations, and pathology. Cambridge, MA: MIT Press.

Giese, M. A., \& Poggio, T. (2003). Neural mechanisms for the recognition of biological movements. Nature Reviews Neuroscience, $\mathbf{4}$, 179-192.

Goldstein, S. R., \& Young, C. A. (1996). "Evolutionary" stable strategy of handedness in major league baseball. Journal of Comparative Psychology, 110, 164-169.

Goodale, M. A., \& HafFenden, A. M. (1998). Frames of reference for perception and action in the human visual system. Neuroscience \& Biobehavioral Reviews, 22, 161-172.

Grondin, S., Guiard, Y., Ivry, R. B., \& Korens, S. (1999). Manual laterality and hitting performance in major league baseball. Journal of Experimental Psychology: Human Perception \& Performance, 25, 747-754.

Grossman, E., Donnelly, M., Price, R., Pickens, D., Morgan, V., NeIghbor, G., \& BlaKe, R. (2000). Brain areas involved in perception of biological motion. Journal of Cognitive Neuroscience, 12, 711-720.

Grouios, G. (2004). Motoric dominance and sporting excellence: Training versus heredity. Perceptual \& Motor Skills, 98, 53-66.

Grouios, G., Koidou, I., Tsorbatzouidis, H., \& Alexandris, K. (2002). Handedness in sport. Journal of Human Movement Studies, 43, 347-361

Grouios, G., Tsorbatzoudis, H., Alexandris, K., \& Barkoukis, V. (2000). Do left-handed competitors have an innate superiority in sports? Perceptual \& Motor Skills, 90, 1273-1282.

GUIARD, Y. (1987). Asymmetric division of labor in human skilled bimanual action: The kinematic chain as a model. Journal of Motor Behavior, 19, 486-517.

Hagemann, N., \& Strauß, B. (2006). Perzeptive Expertise von Badmintonspielern [Perceptual expertise of badminton players]. Zeitschrift für Psychologie, 214, 37-47.

Hagemann, N., Strauß, B., \& Cañal-Bruland, R. (2006). Training perceptual skill by orienting visual attention. Journal of Sport \& Exercise Psychology, 28, 143-158.

Holtzen, D. W. (2000). Handedness and professional tennis. International Journal of Neuroscience, 105, 101-119.

Hommel, B., Müsseler, J., Aschersleben, G., \& Prinz, W. (2001). 
The theory of event coding (TEC): A framework for perception and action planning. Behavioral \& Brain Sciences, 24, 849-878.

Jacobs, A., Pinto, J., \& Shiffrar, M. (2004). Experience, context, and the visual perception of human movement. Journal of Experimental Psychology: Human Perception \& Performance, 30, 822-835.

Johansson, G. (1973). Visual perception of biological motion and a model for its analysis. Perception \& Psychophysics, 14, 201-211.

KNOBLICH, G., \& Flach, R. (2001). Predicting the effects of actions: Interactions of perception and actions. Psychological Science, 12, 467-472.

Loula, F., Prasad, S., Harber, K., \& Shiffrar, M. (2005). Recognizing people from their movement. Journal of Experimental Psychology: Human Perception \& Performance, 31, 210-220.

MaRR, D., \& NishiHARA, H. K. (1978). Representation and recognition of the spatial organization of three-dimensional shapes. Proceedings of the Royal Society of London B, 200, 269-294.

McLean, J. M., \& CiurCZAK, F. M. (1982). Bimanual dexterity in major league baseball players: A statistical study. New England Journal of Medicine, 307, 1278-1279.

McMorris, T., \& Colenso, S. (1996). Anticipation of professional soccer goalkeepers when facing right- and left-footed penalty kicks. Perceptual \& Motor Skills, 82, 931-934.

Milner, A. D., \& Goodale, M. A. (1995). The visual brain in action. Oxford: Oxford University Press.

OldFIELD, R. C. (1971). The assessment and analysis of handedness: The Edinburgh Inventory. Neuropsychologia, 9, 97-113.

Olofsson, U., Nyberg, L., \& Nilsson, L.-G. (1997). Priming and the recognition of human motion patterns. Visual Cognition, 4, 373-382.

Oram, M. W., \& Perrett, D. I. (1996). Integration of form and motion in the anterior superior temporal polysensory area (STPa) of the macaque monkey. Journal of Neurophysiology, 76, 109-129.

Pollick, F. E., Fidopiastis, C., \& Braden, V. (2001). Recognising the style of spatially exaggerated tennis serves. Perception, 30, 323-338.

Pollick, F. E., Lestou, V., RYU, J., \& CHO, S.-B. (2002). Estimating the efficiency of recognizing gender and affect from biological motion Vision Research, 42, 2345-2355.

Raymond, M., Pontier, D., Dufour, A. B., \& Møller, A. P. (1996). Frequency-dependent maintenance of left handedness in humans. Proceedings of the Royal Society of London B, 263, 1627-1633.

Rizzolatti, G., \& Craighero, L. (2004). The mirror-neuron system. Annual Review of Neuroscience, 27, 169-192.

Rossi, B., \& ZANI, A. (1986). Differences in hemispheric functional asymmetry between athletes and nonathletes: Evidence from a unilateral tactile matching task. Perceptual \& Motor Skills, 62, 295-300.

Rowe, R. M., \& McKennA, F. P. (2001). Skilled anticipation in realworld tasks: Measurement of attentional demands in the domain of tennis. Journal of Experimental Psychology: Applied, 7, 60-67.

Runeson, S., \& Frykholm, G. (1981). Visual perception of lifted weight. Journal of Experimental Psychology: Human Perception \& Performance, 7, 733-740.

Stone, J. V. (1998). Object recognition using spatiotemporal signatures. Vision Research, 38, 947-951.

Stone, J. V. (1999). Object recognition: View-specificity and motionspecificity. Vision Research, 39, 4032-4044.

Taddei, F., Viggiano, M. P., \& Mecacci, L. (1991). Pattern reversal visual evoked potentials in fencers. International Journal of Psychophysiology, 11, 257-260.

van der Kamp, J., Rivas, F., van Doorn, H., \& Savelsbergh, G. (2008). Ventral and dorsal system contributions to visual anticipation in fast ball sports. International Journal of Sport Psychology, 39, $100-130$.
Verfaillie, K. (1993). Orientation-dependent priming effects in the perception of biological motion. Journal of Experimental Psychology: Human Perception \& Performance, 19, 992-1013.

Viviani, P. (2002). Motor competence in the perception of dynamic events: A tutorial. In W. Prinz \& B. Hommel (Eds.), Common mechanisms in perception and action (pp. 406-442). Oxford: Oxford University Press.

Ward, P., Williams, A. M., \& Bennett, S. J. (2002). Visual search and biological motion perception in tennis. Research Quarterly for Exercise \& Sport, 73, 107-112.

Williams, A. M., Davids, K., \& Williams, J. G. (1999). Visual perception and action in sport. London: Spon.

Wood, C. J., \& Aggleton, J. P. (1989). Handedness in "fast ball" sports: Do left-handers have an innate advantage? British Journal of Psychology, 80, 227-240.

\section{NOTES}

1. It should be pointed out here that many studies define handedness in terms of the hand used to employ the sports implement in question. This information is usually obtained from current ranking lists that are often accessible to the general public from the Internet. A few studies also determine handedness with standardized questionnaires (see, e.g. Wood \& Aggleton, 1989). This means that in some types of sports, it is unclear whether participants are consistent left-handers who perform all manual activities with their left hand or use the left hand only for that particular sport. It is also impossible to estimate to what extent the preferred hand is influenced by tactical considerations. In baseball, for example, it is common practice to change batting sides because of righthanded pitching or to be closer to first base (e.g., Grondin et al., 1999; McLean \& Ciurczak, 1982). Nonetheless, there are two good reasons for retaining the term left-hander in sport: It is not just the usual term in the literature, it also underlines the fact that the definition refers to the preferred side in one specific type of sport alone. Another assumption, of course, is that there is a high level of agreement between the preferred side in sport and that used in the manual activities listed in standardized questionnaires (see Note 2).

2. Individuals with a negative laterality quotient (LQ; Oldfield, 1971) were identified as left-handers; those with a positive LQ, as righthanders. One participant's playing hand in tennis did not match the outcome of the EHI (Oldfield, 1971). Because he played tennis with the left hand but was right-handed according to the EHI, he was dropped from the data set, and a further participant was recruited for this group (the experts).

3. When the radial error was broken down into the two elements of horizontal error (horizontal deviation) and vertical error (length of stroke), the horizontal deviation revealed a slight tendency toward the backhand side. In other words, the participants believed that the ball would land farther toward the backhand side than it actually did (experts $=11.8 \mathrm{~cm}$, intermediates $=22.0 \mathrm{~cm}$, novices $=27.5 \mathrm{~cm}$ ). The vertical error revealed that the participants tended to estimate that the balls would travel farther than they actually did (experts $=82.3 \mathrm{~cm}$, intermediates $=102.0 \mathrm{~cm}$, novices $=40.3 \mathrm{~cm}$ ). Hence, they tended to expect longer strokes that would also land more to the backhand side. This preference corresponds to the common tactical practice in tennis of playing the longest strokes to the weaker backhand side whenever possible.

(Manuscript received March 18, 2008; revision accepted for publication April 9, 2009.) 